OPEN ACCESS

Edited by:

Johannes Gräff,

EPFL, Switzerland

Reviewed by:

Amy Pooler,

Nestlé Institute of Health Sciences,

Switzerland

Jose Vicente Sanchez-Mut,

EPFL, Switzerland

*Correspondence:

Kobi Rosenblum

kobir@psy.haifa.ac.il

${ }^{\dagger}$ These authors have contributed equally to this work.

Received: 06 April 2016 Accepted: 24 August 2016 Published: 05 September 2016

Citation:

Segev Y, Livne A, Mints $M$ and Rosenblum K (2016) Concurrence of

High Fat Diet and APOE Gene Induces Allele Specific Metabolic and Mental Stress Changes in a Mouse Model of Alzheimer's Disease.

Front. Behav. Neurosci. 10:170. doi: 10.3389/fnbeh.2016.00170

\section{Concurrence of High Fat Diet and APOE Gene Induces Allele Specific Metabolic and Mental Stress Changes in a Mouse Model of Alzheimer's Disease}

\author{
Yifat Segev ${ }^{1 \dagger}$, Adva Livne ${ }^{1 \dagger}$, Meshi Mints ${ }^{1}$ and Kobi Rosenblum ${ }^{1,2 *}$ \\ ${ }^{1}$ Sagol Department of Neurobiology, University of Haifa, Haifa, Israel, ${ }^{2}$ Center for Gene Manipulation in the Brain, University \\ of Haifa, Haifa, Israel
}

Aging is the main risk factor for neurodegenerative diseases, including Alzheimer's disease (AD). However, evidence indicates that the pathological process begins long before actual cognitive or pathological symptoms are apparent. The long asymptomatic phase and complex integration between genetic, environmental and metabolic factors make it one of the most challenging diseases to understand and cure. In the present study, we asked whether an environmental factor such as high-fat (HF) diet would synergize with a genetic factor to affect the metabolic and cognitive state in the Apolipoprotein $E$ (ApoE4) mouse model of AD. Our data suggest that a HF diet induces diabetes mellitus (DM)-like metabolism in ApoE4 mice, as well as changes in $\beta$-site amyloid precursor protein-cleaving enzyme 1 (BACE1) protein levels between the two ApoE strains. Furthermore, HF diet induces anxiety in this AD mouse model. Our results suggest that young ApoE4 carriers are prone to psychological stress and metabolic abnormalities related to $A D$, which can easily be triggered via HF nutrition.

Keywords: ApoE4, metabolic stress, high-fat diet, Alzheimer's disease (AD), ATF4, translation regulation

\section{INTRODUCTION}

Aging is the main risk factor for Alzheimer's disease (AD), which is a complex multifactorial disease involving both genetic and environmental factors. AD affects about 40 million people worldwide, with predictions for a dramatic increase in the coming years with the increase in the aged population (Dacks et al., 2014).

The most common form of the disease, sporadic, late onset $\mathrm{AD}$, strikes on average after the age of 65, and is strongly linked to the Apolipoprotein E (ApoE) gene on chromosome 19. ApoE encodes a 299 -amino acid, $34-\mathrm{kDa}$ glycoprotein most prevalent in the liver, followed by the brain. ApoE mediates the transport and clearance of cholesterol, triglycerides, and other lipids (Li et al., 1988). The three human ApoE isoforms $\varepsilon 2, \varepsilon 3$ and $\varepsilon 4$ differ from one another at residues 112 and 158, affecting their 3 -dimensional structure. The $\varepsilon 4$ allele has approximately $20 \%$ frequency in the general pooled population and roughly $50 \%$ in patients with $\mathrm{AD}$, however, there is substantial heterogeneity in these prevalence estimates (Ward et al., 2012). The above finding renders ApoE4 the major genetic risk factor for sporadic 
AD (Rall et al., 1982; Strittmatter and Roses, 1996; Ward et al., 2012).

Different pathological mechanisms have been proposed to explain the effect of ApoE4, including mainly defects in the clearance of the beta-amyloid $(\mathrm{A} \beta)$ peptide, lipid and cholesterol metabolism, inflammation, mitochondrial dysfunction, neuronal function, and to a lesser extent, amyloid precursor protein (APP) cleavage (Mahley and Rall, 2000; He et al., 2007; Castellano et al., 2011; Chen et al., 2012; Dafnis et al., 2012). In addition, diabetes mellitus (DM) was suggested to exacerbate the risk of $\mathrm{AD}$ associated with ApoE4, as patients with diabetes who carry the ApoE4 allele are twofold more likely to develop AD than non-diabetic ApoE4 carriers (Peila et al., 2002). A possible mechanism specifically linking insulin resistance to $\mathrm{AD}$ in $\mathrm{ApoE} 4$ carriers is that $\mathrm{A} \beta$ is in part cleared by the insulin degrading enzyme (IDE). AD patients carrying the ApoE4 allele have been reported to express reduced IDE protein and mRNA levels in the hippocampus (Biessels et al., 2005; Schipper, 2011), suggesting a causal link between impaired insulin metabolism, either hypoinsulinemia or insulin resistance, and the pathogenesis of $\mathrm{AD}$ (Schipper, 2011).

Diverse metabolic stresses such as described above are mirrored in part by changes in the phosphorylation of eukaryotic initiation factor 2 (eIF $2 \alpha)$ at serine 51 in cells and specifically in neurons, leading to changes in translation regulation (Sonenberg et al., 2000). At the same time, inverse correlation has been identified between eIF2 $\alpha$ phosphorylation and memory or synaptic plasticity consolidation (Costa-Mattioli et al., 2007). Several lines of evidence indicate that phosphorylation of eIF2 $\alpha$ or activation of its kinase, double-stranded RNA-activated protein (PKR), are involved in the pathogenesis of $\mathrm{AD}$ (Chang et al., 2002; Page et al., 2006). We have previously shown that ApoE4 AD model mice express high phosphorylation levels of eIF $2 \alpha$ in the hippocampus compared to their age matched ApoE3 controls as early as 4 months of age (Segev et al., 2013). Furthermore, this increased phosphorylation is correlated with increased mRNA expression of activating transcription factor4 (ATF4) mRNA both in these mice and in postmortem AD brains (Segev et al., 2015).

Genome-wide association studies identified ApoE4 as the only significant gene associated with age-related cognitive decline in humans (De Jager et al., 2012). Additional studies suggest that ApoE4 has a detrimental effect on cognition before the typical signs of $\mathrm{AD}$ are apparent (Caselli et al., 2009). Supporting the above is the fact that we and others have shown that glial fibrillary acidic protein (GFAP)-ApoE4 expressing human-ApoE4 in astrocytes under the control of a GFAP promoter, as well as transgenic hAPP-ApoE4 and human-ApoE4 mice carrying the human ApoE4 gene under the endogenous ApoE mouse promoter, are impaired in spatial memory compared with their ApoE3 controls (Raber et al., 2000; Hartman et al., 2001; Kornecook et al., 2010; SalomonZimri et al., 2014; Segev et al., 2015). However, another study (Olsen et al., 2012) showed no memory deterioration in human-ApoE4 mice in the contextual memory of Fear Conditioning. These conflicting results may be due to the different mouse strains, but more likely due to the different protocols used. While Olsen and colleagues used a relatively strong protocol of two shocks, we used a single shock paradigm (Olsen et al., 2012; Segev et al., 2013). In our experience, a stronger shock makes it hard to detect cognitive differences between the mouse strains at a young age, therefore to detect changes in memory one needs to use a weaker protocol.

An additional factor linking metabolic stress to $\mathrm{AD}$, is the $\beta$-site APP-cleaving enzyme 1 (BACE1). BACE1 is the initiator enzyme for the formation of $A \beta$, and surprisingly, increased phosphorylation of eIF $2 \alpha$ as a result of metabolic stress, while decreasing general protein synthesis, increases a subset of mRNAs such as ATF4 (Costa-Mattioli et al., 2007) and BACE1 (O'Connor et al., 2008). Indeed, increased BACE1 protein levels and activity have been reported in the brains from $\mathrm{AD}$ patients (Fukumoto et al., 2002). In addition, BACE1 has been studied in relation to insulin deficiency and obesity. For example, a study by Devi et al. (2012) suggested that translational mechanisms through phosphorylation of eIF $2 \alpha$ may underlie the upregulation of BACE1 associated with insulin deficient diabetes. Another group showed that mice lacking BACE1 are lean, resistant to dietinduced obesity and display increased peripheral tissue insulin sensitivity with improved whole-body glucose disposal (Meakin et al., 2012).

In addition to the known symptoms of cognitive impairment, increased anxiety may occur in up to $70 \%$ of $\mathrm{AD}$ patients during the course of their illness (Ferretti et al., 2001), and specifically patients with $\epsilon 4 / \epsilon 4$ show higher anxiety scores than those with $\epsilon 3 / \epsilon 3$ (Robertson et al., 2005).

In the present study, we tested the hypothesis that a high-fat (HF) diet converges on the ApoE4 allele, resulting in enhanced attenuation of cognitive abilities and metabolic phenotype. In order to test this hypothesis, we provided humanApoE4 transgenic mice, which express the human ApoE gene physiologically under the murine ApoE promoter, and their ApoE3 controls with a HF diet for 20 weeks post- weaning, after which they were evaluated for metabolic and behavioral phenotypes. In agreement with others, we show that although ApoE4 mice gain less weight over time (Huebbe et al., 2015), they develop DM-like features (Arbones-Mainar et al., 2008; Pendse et al., 2009). Interestingly, consumption of the HF diet induces higher anxiety levels in the HF-apoE4 mice compared with HF-ApoE3 controls. Furthermore, BACE1 protein levels are increased in the hippocampus of HF-ApoE4 mice. Our findings point out the importance of convergence of environmental and genetic factors in the etiology and progression of $\mathrm{AD}$ at a young age, before pathological symptoms are apparent, and propose for further pursuing this hypothesis for additional and broader studies.

\section{MATERIALS AND METHODS}

\section{Animals}

Humanized knock-in ApoE3 (B6.129P2-ApoE ${ }^{\text {tm2(ApoE*3)Mae }}$ N8) and knock-in ApoE4 (B6.129P2-ApoEtm3(ApoE*4)Mae N8) 
homozygous male mice were supplied by Taconic (Hudson, NY, USA). All cages were placed in a light and temperature controlled room, and behavioral tests were conducted during daylight hours. All animals were handled in accordance with the University of Haifa regulations and the National Institutes of Health Guidelines (NIH publication number 8023), and maintained in a pathogen-free environment.

\section{Animal Treatment}

All animal care and procedures were carried out in accordance with local guidelines and approved by the Animal Care and Use Committee of the University of Haifa. All efforts were made to minimize animal suffering and discomfort.

One month old male mice, both ApoE3 and ApoE4, were weaned, group housed in cages consisting of two or three littermates and given immediately a diet consisting of $60 \%$ fat ("high fat diet", TD.06414, Harlan, WI, USA) ad libitum for a total of 3 months. Animals were weighed using lab scales and food intake was inspected on a weekly basis as the difference in mass between what was given at feeding time and the amount that was left 1 week later. The same animals were used for all experiments to assess the influence of the HF diet on the behavior of the different strains. Animals were sacrificed by cervical dislocation 2 weeks after the restraint test.

\section{Metabolic Measurements and Plasma Collection}

Peripheral blood glucose was measured using an Accu-chek Performa glucometer (Roche, Switzerland) after nicking the tail tip. Non-fasting glucose levels were measured between 10 and 11:30 AM. Mice were deprived of food overnight for $12 \mathrm{~h}$ for fasting insulin measurements. Peripheral blood for insulin was collected by cardiac puncturing, after which mice were sacrificed. Blood was then diluted in $0.5 \mathrm{M}$ EDTA and centrifuged for $25 \mathrm{~min}$ at $4^{\circ} \mathrm{C}$ and $3500 \mathrm{RPM}$. Plasma Insulin was determined using Rat/mouse Insulin Elisa kit \#EZRMI-13K (Merck Millipore, Germany) according to the manufacturer's instructions.

\section{Fear Conditioning}

Fear conditioning was conducted in model chambers, measuring $(25 \times 25 \times 25) \mathrm{cm}$ internally (Panlab Harvard Apparatus, Barcelona, Spain). Each chamber was located inside a larger, insulated plastic cabinet that excluded external light and noise. The system enabled recording and analysis of the signal generated by animal movement via a high-sensitivity weight transducer system. Mice were placed in a chamber (context A with light [20 W bulb] and a 16-bar metal grid floor) for $120 \mathrm{~s}$, after which the mice received a $2.9 \mathrm{kHz}$ tone, applied for $30 \mathrm{~s}$ at $80 \mathrm{~dB}$ (conditioned stimuli; CS) and a subsequent $0.6 \mathrm{~mA}$ shock applied for $2 \mathrm{~s}$ (unconditioned stimuli; US), after which they remained in the chamber for 60 additional seconds and were returned to their home cage. The chambers were cleaned with $10 \%$ ethanol between successive sets of mice. Animal behavior was recorded, and the data were analyzed by Freeze Frame 3.0 software (Coulbourn Instruments, Whitehall, PA, USA).
The indication for fear memory was percentage of time spent freezing.

\section{Elevated Plus Maze}

The elevated plus maze (EPM) paradigm was conducted in a standard plus maze. The maze apparatus consisted of four arms in a cross position from a neutral central square. The horizontal arms are defined as "closed arms" $(52 \mathrm{~cm} \times 7 \mathrm{~cm} \times 15 \mathrm{~cm})$ and the vertical arms are defined as "open arms" and have no protective walls $(52 \mathrm{~cm} \times 7 \mathrm{~cm}$ ). The $50 \mathrm{~cm}$ high maze was placed on a table in a room with indirect light. At the beginning of a 10 min test session, each mouse was placed in the middle of the maze facing the open arms and movement was monitored and registered by a camera system connected to an Ethovision XT system (Noldus, Leesburg, VA, USA). Time in closed arms was expressed as the percentage of time spent in the closed arms during the duration of the test and body elongation as the cumulative time the mouse stretched at least half of its body from a standing position at the center to the area of the open arms. The maze was cleaned with $10 \%$ ethanol between successive tests.

\section{Tissue Extraction}

Mice were injected subcutaneously with ketamine $(0.01 \mathrm{~mL} / \mathrm{g})$ and were decapitated after cervical dislocation. Brains were removed rapidly and frozen using liquid nitrogen. Coronal sections were cut on a Leica CM3050S cryostat using RichardAllan Sec5e blades until the amygdala was exposed and was dissected from the caudal side. Sections were taken further on until the hippocampus area was exposed and dissected similarly. Single or multiple punches were taken to remove the area in discussion. Hippocampal punches removed the entire hippocampus, and amygdala punches removed the basolateral (BLA) area. Punches for each area from both hemispheres were pooled and stored together for each mouse in the same tube, then stored at $-80^{\circ} \mathrm{C}$ until protein level evaluation.

\section{Western Blotting}

\section{Preparation of Cell Extracts}

All protein levels were evaluated in hippocampal lysates by Western blot analysis. Lysates were made by homogenizing samples in SDS sample buffer. A volume of $15 \mu \mathrm{l}$ of each sample was loaded into its respective well in a 26 well Criterion TGX gel (Bio-Rad, Hercules, CA, USA). Every two gels were placed in a Criterion electrophoresis cell (Bio-Rad, CA, USA) and electrophoresed under a $200 \mathrm{~mA}$ current for $90 \mathrm{~min}$. Transfer was done using the Trans-Blot Turbo Transfer system (Bio-Rad, Hercules, CA, USA) onto $0.45 \mu \mathrm{m}$ nitrocellulose membranes (Bio-Rad, Hercules, CA, USA). Blots were blocked in freshly prepared Tris-buffered saline solution containing $0.1 \%$ Tween 20 (TBST) with $2-5 \%$ BSA for $1 \mathrm{~h}$ at room temperature, with agitation. Primary antibodies and preparation: BACE Rabbit mAB \#5606 (Cell Signaling, Danvers, MA, USA) diluted $1: 500$ in $4 \%$ BSA and $0.1 \%$ sodium azide; total-eIF2 $\alpha$ Mouse mAB \#2103 (Cell Signaling, MA, USA) 
diluted 1:1000 in 4\% BSA and $0.1 \%$ sodium azide; phosphoeIF2 $\alpha$ Rabbit mAB \#3398 (Cell Signaling, Danvers, MA, Danvers, USA) diluted $1: 1000$ in $4 \% \mathrm{BSA}$ and $0.1 \%$ sodium azide.

Each membrane was incubated overnight with its respective primary antibody, then washed in TBST three times for $5 \mathrm{~min}$ each. Following this series of washing steps, membranes were left for an hour incubation with a secondary antibody. Secondary antibodies were prepared in TBST solution and according to the specific primary antibody. BACE and p-eIF $2 \alpha$ membranes were incubated in $\alpha$ Rabbit antibody $(1: 10,000)$, whereas eIF2 $\alpha$ membrane was incubated in $\alpha$ Mouse antibody $(1: 10,000)$. Membranes were then developed using an XRS camera and the data analyzed by Quantity one 1-D Analysis software (Bio-Rad, Hercules, CA, USA).

\section{Real Time mRNA}

Total RNA (1 $\mu g$ ) extraction from the amygdala was performed using the RNAeasy Lipid Tissue mini kit (QIAGEN, Netherlands) according to the manufacturer's recommendations. Reverse transcription was made using the Invitrogen Superscript III first strand synthesis (Live technologies, Carlsbad CA, USA). RNA samples were mixed with random hexamer primers and were incubated at $65^{\circ} \mathrm{C}$ for $5 \mathrm{~min}$ and briefly centrifuged. The samples then underwent a reaction using $10 \mu \mathrm{l}$ reaction mix and then incubated again as follows: $5 \mathrm{~min}$ in $25^{\circ} \mathrm{C}$ then $50^{\circ} \mathrm{C}$ for $50 \mathrm{~min}$. The reaction was terminated at $85^{\circ} \mathrm{C}$ for $5 \mathrm{~min}$ and was stored at $-20^{\circ} \mathrm{C}$. Real time RT-PCR was performed in triplicates using the ABI PRISM StepOne plus Sequence Detector (Life technologies, Grand Island, NY, USA). The analyzed genes were corticotropin releasing factor (CRF) primer \#205769, CRF1 primer \#7762, and NR3c1 primer \#8173, all from the TaqMan Gene Expression Assays primers (Life Technologies, Grand Island, NY, USA) according to existing literature.

\section{Statistical Analysis}

Statistical analyses were performed using GraphPad Prism6. Means of two groups were compared with a two-tailed $t$ test or the Mann-Whitney-Wilcoxon U test. Comparisons of multiple groups or measurements were analyzed with one-way analysis of variance (ANOVA) or two-way ANOVA, followed by the appropriate post hoc tests. All groups were initially checked for normal distribution of data before proceeding with the analysis.

\section{RESULTS}

\section{High-Fat Diet Induces Changes in Anxiety Behavior Between ApoE3 and ApoE4 Mice}

In this study, we hypothesized that consumption of a HF diet would differentially affect ApoE4 vs. ApoE3 mice cognitively, i.e., it would further increase their metabolic burden, and as a consequence, increase differences observed in the behavioral phenotype of the hippocampal-dependent contextual memory between the two strains. For this purpose, ApoE4 $(n=21)$ and ApoE3 $(n=20)$ mice in two different batches pooled for analysis were fed on a HF diet (60\% fat) for 20 weeks from weaning age. At the end of this time period the mice were tested for behavioral performance in the fear conditioning paradigm. This test was initially chosen because it had the strongest phenotype effect, however, unexpectedly, the mice showed significant differences as early as the conditioning phase of the foot shock (Figure 1A). HF-ApoE4 mice showed significantly higher freezing levels than HF-ApoE3 controls (2-way ANOVA repeated measures; $F_{(1,117)}=33.01$, **** $P<0.0001)$.

These intriguing results prevented us from further testing our initial hypothesis and prompted us to propose a new one: HF diet differentially affects stress-like behavior in HFApoE3 vs. HF-ApoE4 mice as indicated in the higher freezing levels of HF-ApoE4 mice after presentation of the US alone. In order to test differential anxiety behavior in the two experimental groups, we used the EPM paradigm. Mice were placed on a $50 \mathrm{~cm}$ high plus maze placed on a table, with two open and two closed arms for a total exploration time of $10 \mathrm{~min}$, during which their behavior was recorded. HF-ApoE4 $(n=10)$ mice spent significantly more time in the closed arms compared to HF-ApoE3 $(n=8)$ mice, reminiscent of stressful behavior (Hogg, 1996; Figure 1B; Mann Whitney test, $\left.{ }^{*} p=0.0341\right)$. There were no differences in movement/activity between the two mouse strains (data not shown).

A more detailed analysis of behavior revealed differences in risk assessment (RA) between the two mouse strains (Blanchard et al., 1990; Griebel et al., 1995). HF-ApoE4 mice exhibited longer time spent in the stretched attended posture (SAP) compared to HF-ApoE3 mice (Figure 1C; Unpaired $t$-test, $t_{(16)}=2.931$, $\left.{ }^{* *} p=0.0098\right)$, mainly at time points of aborted attempts of entry into the open arms. RA refers to a pattern of responses such as scanning, stretch attended posture, and flat back approaching (Griebel et al., 1997).

\section{HF-ApoE4 Mice do not Express More Stress-Induced Plasticity in the Amygdala Compared to HF-ApoE3 Controls}

We next asked whether the anxiety behavior seen in young ApoE4 mice fed on the HF diet is reflected by differences in expression levels of stress-related neuropeptide mRNA in the amygdala. The CRF peptide system is critical for the behavioral response to stressful situations such as anxiety in vertebrates (Bale and Vale, 2004; Hauger et al., 2006). We thus examined the amygdala of HF-ApoE4 $(n=10)$ and HF-ApoE3 $(n=8)$ mice for CRF, CRF receptor type1 (CRF1), and glucocorticoid receptor (NR3C1) mRNA levels. We did not detect any significant changes in the above factors between the two mouse strains (Figure 2A).

We have previously shown that young (4 month) naïve ApoE4 mice express high ATF4 mRNA levels in the hippocampus compared with ApoE3 controls (Segev et al., 2015). Here, we observed that this increase is also apparent in the amygdala of the mice fed on the HF diet (Figure 2A; Unpaired t-test, $\left.t_{(15)}=2.831,{ }^{*} p=0.0126\right)$, suggesting that the increased 
A

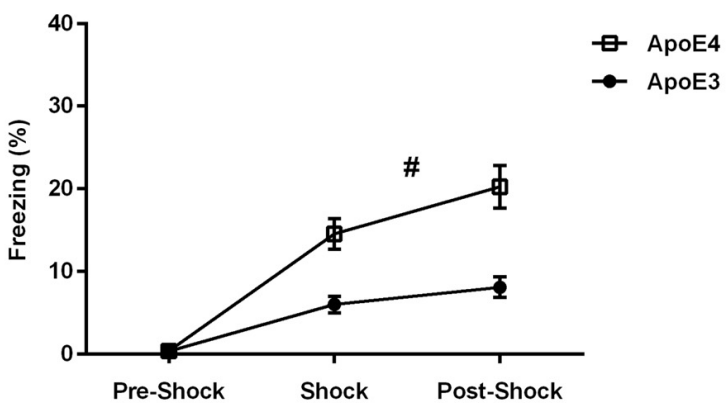

B

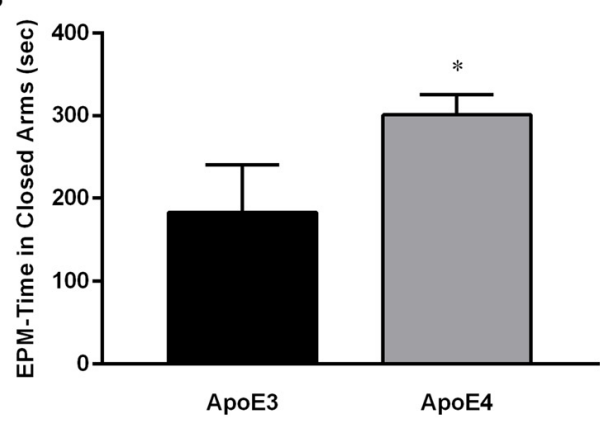

C

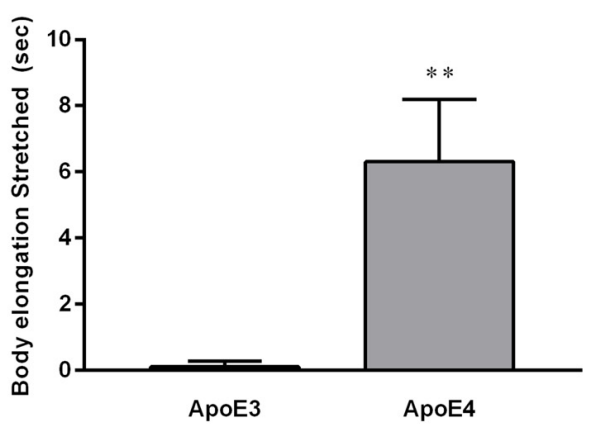

FIGURE 1 | High-fat (HF) diet induces changes in anxiety behavior between Apolipoprotein E (ApoE3) and ApoE4 mices. (A) HF-ApoE4 and HF-ApoE3 mice were placed in an isolated chamber and after $60 \mathrm{~s}$ were introduced with a $2 \mathrm{~s} 0.5 \mathrm{~mA}$ foot shock followed by a 60 s long post-shock interval. HF-ApoE4 mice showed higher freezing time after the presentation of the foot shock, compared to HF-ApoE3 mice (Two-Way repeated measures analysis of variance (ANOVA), $F_{(1,39)}=17.06$, $\# p=0.0002$ ). (B) Four to five months old ApoE4 mice and age matched ApoE3 controls that consumed a HF diet for 20 weeks were placed in an elevated plus maze (EPM) for a period of $10 \mathrm{~min}$, while their behavior was evaluated. HF-ApoE4 mice spent a longer time in the closed arms of the EPM $(300.7 \pm 24.37, n=10)$ compared with ApoE3 mice (183.0 $\pm 57.18 n=8$; Mann-Whitney test, $\left.{ }^{*} p=0.0341\right)$.

(C) HF-diet mice were evaluated for cumulative duration of body elongation stretched posture. ApoE4 mice displayed a significantly longer

( $6.304 \pm 1.880, n=10)$ period of time in the stretched posture, compared to ApoE3 mice $\left(0.1050 \pm 0.05803, n=8 ; t_{(16)}=2.931,{ }^{* *} p=0.0098\right)$. Data are shown as the mean \pm SEM. "Relates to the repeated measuring effect of freezing between the groups $p<0.05$.

expression levels of ATF4 in the central nervous system (CNS) of ApoE4 mice are not specific to the hippocampus. We cannot conclude from this, however, that the increased
A

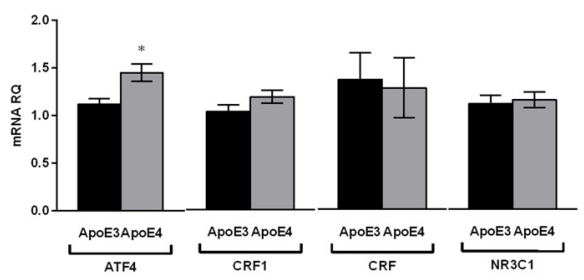

B

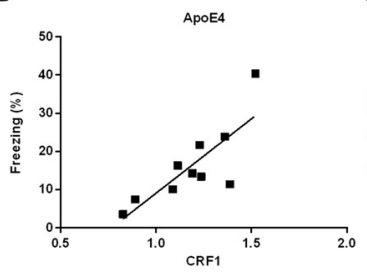

C

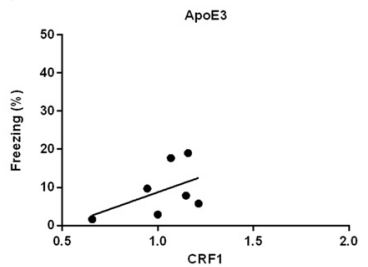

FIGURE 2 | HF-ApoE4 do not express stress-induced plasticity in the amygdala compared to HF-ApoE3 mice, but display correlation between corticotropin releasing factor 1 (CRF1) expression and freezing percent post foot-shock. Whole amygdala was punched from the brains of mice maintained on a HF diet, and mRNA was extracted for evaluation of specific mental stress-related genes as well as the metabolic stress-related gene activating transcription factor 4 (ATF4). (A) There were no significant differences between HF-ApoE4 and HF-ApoE3 mice in CRF1 $\left(t_{(15)}=1.554, p=0.1411\right), \operatorname{CRF}\left(t_{(15)}=0.1911, p=0.8510\right)$, and NR3C1 $\left(t_{(15)}=0.3328, p=0.7439\right)$. ATF4 expression was higher in HF-ApoE4 (1.449 $\pm 0.08855, n=10)$ compared to HF-ApoE3 mice (1.116 \pm 0.05892 , $\left.n=7 ; t_{(15)}=2.831,{ }^{*} p=0.0126\right)$. (B,C) Correlations; (B) HF-ApoE4 mice displayed significant correlation between amygdala CRF1 mRNA and foot shock-dependent freezing percent $\left(p=0.0052, R^{2}=0.6446\right)$. (C) However, there was no significant correlation in HF-ApoE3 mice between amygdala CRF1 mRNA and freezing percent $\left(p=0.2656, R^{2}=0.2389\right)$. Correlation was analyzed using Pearson $r$ test. Data are shown as the mean \pm SEM.

expression levels in the amygdala are due to the consumption of the HF diet, since the analysis was not performed on the amygdala of naive mice. Interestingly, although there were no significant differences in CRF1 mRNA levels between the two mouse strains, HF-ApoE4 mice showed a strong and significant correlation between CRF1 levels and the freezing percent in response to the foot shock presentation (Figure 2B; Pearson $\left.r=0.802,{ }^{* *} p=0.0052\right)$, whereas HF-ApoE3 mice did not show this tendency (Figure 2C; Pearson $r=0.488$, $p=0.2656$ ), suggesting that the anxiety behavior in HF-ApoE4 mice is coordinated, at least in part, by CRF receptors in the amygdala.

\section{ApoE4 Mice Develop Diabetes Mellitus-like Metabolic Features Following Maintenance on a High-Fat Diet}

Dysregulation of glucose and insulin homeostasis as well as obesity are risk factors for the development of $\mathrm{AD}$ (Ho et al., 2004; de la Monte and Wands, 2008; Maesako et al., 2015). Furthermore, there is growing evidence in support of the hypothesis that $\mathrm{AD}$ represents a form of $\mathrm{DM}$ that selectively affects the brain (de la Monte and Wands, 2008). Here, we confirm previous work by others (Huebbe et al., 2015), showing that ApoE4 mice fed on a HF diet gain less weight over 
time, compared with their HF-ApoE3 age matched controls (Figure 3A; 2-way repeated measures ANOVA, strain effect; $\left(F_{(1,16)}=13.54,{ }^{* *} p=0.0020\right)$.

We further demonstrate that consumption of a HF diet alone is sufficient to cause DM-like metabolic features apparent by disruption in peripheral glucose and insulin levels in the HFApoE4 AD mouse model (Figures 3B,C). Specifically, HF-ApoE4 $(n=10)$ mice have lower levels of peripheral plasma insulin after a $24 \mathrm{~h}$ starvation period $\left(t_{(15)}=4.643,{ }^{* * *} P=0.0003\right)$, and higher glucose levels $\left(t_{(15)}=2.924,{ }^{*} P=0.0105\right)$ compared with HF-ApoE3 $(n=8)$ controls (Figure 3D). We asked whether the glucose and insulin levels in the HF-ApoE4/3 mice resemble those of C57BL/6 mice fed with the same diet (data obtained from Harlan laboratories), which would suggest whether the changes are specific to the $\varepsilon 4$ allele. C57BL/6 mice fed with this diet for a period of 17 weeks show glucose levels of $146 \mathrm{mg} / \mathrm{dl}$. Both HF-ApoE3 and HF-ApoE4 show higher levels of glucose, however, ApoE4 mice show higher levels $(287 \mathrm{mg} / \mathrm{dl})$ compared to ApoE3 mice. This hypothesis should be further evaluated by comparing the data to groups of ApoE3 and E4 fed on a normal diet. Interestingly, HF-ApoE4 mice showed a stronger correlation between body weight and peripheral serum insulin levels (Pearson $r,{ }^{* *} P=0.0016$ ) than HF-ApoE3 mice (Pearson $r$, $\left.{ }^{*} P=0.033\right)$. Differences between elevations are highly significant $\left({ }^{* * *} p=0.0003657\right)$.

\section{High-Fat Diet Promotes Changes in BACE1 Protein Expression in the Hippocampus Between ApoE3 and ApoE Mice}

We have previously shown that phosphorylation of eIF $2 \alpha$ is increased in 4 month old ApoE4 mice (Segev et al., 2015). As observed before in naïve mice, HF-ApoE4 mice express higher levels of eIF $2 \alpha$ phosphorylation in the hippocampus compared with HF-ApoE3 controls (Figure 4A; $t_{(27)}=6.367$, **** $P<0.0001)$. In addition, HF-ApoE4 $(n=10)$ mice express higher BACE1 protein levels in the hippocampus compared to HF-ApoE3 $(n=8)$ controls (Figure $4 \mathbf{B} ; t_{(25)}=2.204$, $\left.{ }^{*} P=0.0369\right)$.

Work by others suggests that the increase in BACE1 levels may be related to the HF consumption and the DM-like metabolic features (Patil et al., 2006; Zhang et al., 2009; Son et al., 2012), however, it has never been shown, to the best of our knowledge, in the ApoE4 AD model. There was no correlation between hippocampal BACE1 protein levels and ATF4 mRNA levels in the amygdala. This can be explained by the different measurements, i.e., mRNA vs. protein, the different tissues analyzed, i.e., amygdala vs. hippocampus, and the fact that the differences between the elevations are highly significant (Figure 4C; $\left.F_{(1,13)}=13.4541,{ }^{* *} P=0.0028\right)$.

\section{DISCUSSION}

In agreement with the early emergence and slow progression of $\mathrm{AD}$, environmental factors are becoming more important in our consideration of ways to delay the onset of disease symptoms. Nutrition is one such environmental factor, which is closely related to both cholesterol metabolism, i.e., ApoE genotype, and insulin/glucose impaired metabolism, i.e., DM, which are both risk factors for $\mathrm{AD}$.

We have previously demonstrated that young humantargeted ApoE4 replacement mice show mild cognitive impairment (MCI) in the contextual fear conditioning paradigm, compared with ApoE3 age matched controls (Segev et al., 2013). Later publications by other groups strengthened these findings of cognitive deficiency in this mouse strain (Salomon-Zimri et al., 2014). In our previous experiments with standard diet fed mice, basal freezing levels in the conditioning phase were consistently similar between the two groups, suggesting that the unconditioned stimulus, foot shock, by itself did not cause behavioral/anxiety differences between the groups. In the present study, we asked whether a simple, noninvasive intervention such as a western-like HF diet would have an effect on the metabolism and/or cognitive behavior of an AD-relevant mouse model, the ApoE4 mice. We initially hypothesized that the cognitive impairments in young naive ApoE4 mice (Segev et al., 2013) would be worsened by a HF diet. To this end, the AD-relevant model mice and their controls, ApoE3 mice, were given a western-like HF diet from weaning age until they reached 4 months, the age in which we previously detected behavioral abnormalities and eIF2 $\alpha$ increased phosphorylation (Segev et al., 2013). However, unexpectedly, ApoE4 mice displayed higher anxiety levels as early as the presentation of a foot shock on the conditioning day of the fear conditioning paradigm. Due to these results the mice were not further evaluated for the memory of the fear conditioning. Instead, they were analyzed for anxiety in the EPM. The results suggest that expression of human-ApoE4 allele renders mice fed on a western-like diet more susceptible to mental stress. It should be noted that the SAP differences in behavior observed here do not seem to be necessarily related to the induction of a HF diet, since a work presented by Hartman et al. (2001) showed that 8-11 month old GFAP-ApoE4 mice fed on a normal diet do not display significant differences in the time spent in the different arms. However, these mice possess higher SAP time compared with GFAP-ApoE3 mice (Hartman et al., 2001). The results described in this study and our own suggest that ApoE4 mice have low basal levels of anxiety, which are apparent only in the SAP. However, the ApoE4 mice are prone to mental stress that can easily be triggered by environmental stimuli such as consumption of a HF diet, as indicated by higher freezing levels after presentation of a foot shock and longer time spent in the closed arms of the EPM. Conflicting evidence is apparent in regard to the level of basal anxiety in ApoE4 mice. While Johnson et al. (2014) showed differences in basal anxiety levels related to the E4 allele, Salomon-Zimri et al. (2014) showed that there were no changes in basal anxiety levels between the ApoE4 and ApoE3 mice fed on a normal diet. This could be explained either by the fact that different protocols were used in the different studies or that the basal anxiety levels are mild in normal-fed mice and therefore may not always be apparent. In any case, our results advocate that the anxiety behavior is worsened with a HF diet specifically in ApoE4 mice. For this reason the same mice (from the second 

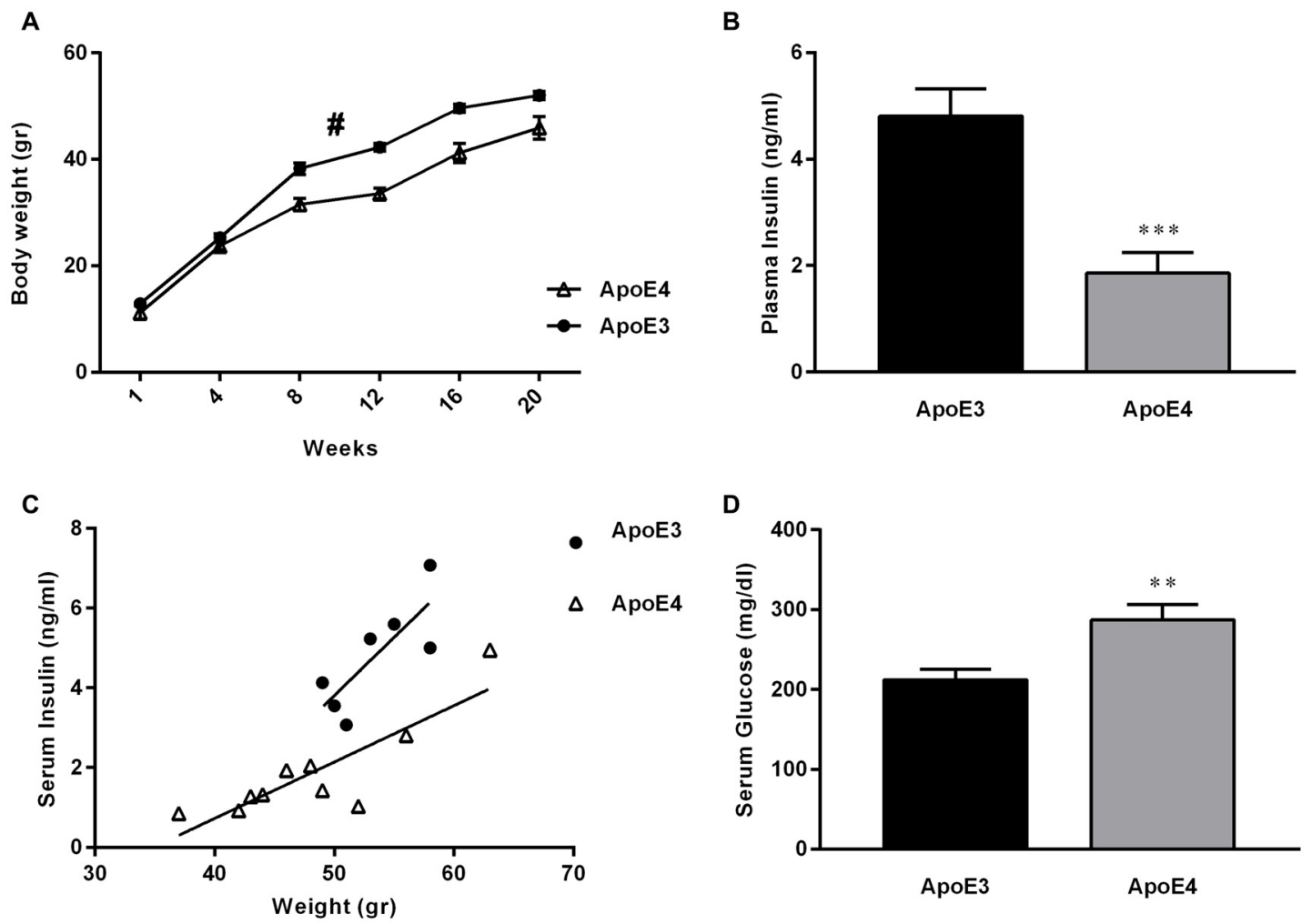

FIGURE 3 | ApoE4 mice develop diabetes mellitus (DM)-like metabolic features following maintenance on a HF diet. (A) ApoE4 mice gained significantly less weight over a HF diet period of 20 weeks compared to ApoE3 mice (Two-way repeated measures ANOVA, strain effect; $F_{(1,16)}=13.54$, $\left.{ }^{*} p=0.0020\right)$. (B) Serum-insulin was extracted and evaluated from $24 \mathrm{~h}$ fasted ApoE4 mice and ApoE3 controls after a 23 week HF diet consumption. HF-ApoE4 mice showed significantly lower levels of serum insulin $(1.857 \pm 0.3930, n=10)$, compared with HF-ApoE3 mice $\left(4.809 \pm 0.5133, n=7 ; t_{(15)}=4.643,{ }^{* * *} p=0.0003\right)$.

(C) Correlation between body weight and serum insulin was evaluated. There was a stronger correlation in HF-ApoE4 mice $\left(R^{2}=0.7317\right.$, $\left.{ }^{\star \star} p=0.0016\right)$, compared to HF-ApoE3 mice $\left(R^{2}=0.6297, p=0.0332\right)$. In addition, linear regression test demonstrated significant differences between elevations or intercepts

$\left(F_{(1,14)}=21.7475,{ }^{* * *} p=0.00036\right)$, and no differences between slopes $\left(F_{(1,13)}=2.65066, p=0.1275\right)$. (D) Serum-glucose was extracted from non-fasting ApoE4 and ApoE3 mice after a 20 week HF diet. HF-ApoE4 mice showed significantly higher glucose levels $(287.2 \pm 19.25, n=10)$ compared with HF-ApoE3 age matched controls $\left(212.1 \pm 13.25, n=7 ; t_{(15)}=2.924,{ }^{* *} p=0.0105\right)$. Data are shown as the mean \pm SEM. ${ }^{*}$ Relates to the repeated measuring effect of body weight between the groups $(p<0.05)$. Correlations were analyzed using the Pearson $r$ test.

batch only) were evaluated for anxiety levels using the EPM, and were not further tested for memory differences in the fear conditioning paradigm. It would be vital to evaluate the effect of genotype vs. the effect of a HF on stress behavior as well as performance on additional learning and memory paradigms. This can be done by comparison to additional groups of naïve mice fed on a normal diet. It should also be noted that a study by Siegel et al. (2012) showed that female human-ApoE4 mice express higher measures of anxiety compared to female human-apoE3 controls, and as a result, seemed to score better at the hippocampal-dependent learning of Morris water maze (MWM). On the other hand, another group detected impairments in the MWM learning paradigm in male human-ApoE4 vs. male ApoE3 mice with no apparent anxiety behavior (Salomon-Zimri et al., 2014). These conflicting results may be due to differences in protocols and gender of mice.

Our results show no changes in CRF peptide system mRNAs in the amygdala, however, these were only analyzed in non-stressed HF-ApoE mice. These mRNAs should be evaluated in mice after a stressful stimulus such as the foot shock in order to evaluate whether the stressful behavior is mirrored by changes in the CRF peptide system in the amygdala.

Work by other groups revealed that although ApoE4 mice gain less weight after consumption of a HF diet, they display dysfunctional epididymal adipose tissue, which in turn impairs the glucose tolerance in these mice (Arbones-Mainar et al., 2008). In our work, we strengthen the above by showing that ApoE4 mice indeed gain less weight over time and display abnormal peripheral glucose/insulin levels, suggesting that the $\epsilon 4$ allele together with an imbalanced HF diet is sufficient to induce DMlike features, which in turn may also render ApoE4 carriers more susceptible to dementia and AD (Peila et al., 2002; Yang and Song, 2013). Furthermore, the reduced body weight in the HF-ApoE4 mice may be in part due to impaired insulinstimulated growth and survival signaling. The fact that ApoE4 mice show stronger correlation between body weight and insulin levels suggests impaired ability to balance insulin levels despite 

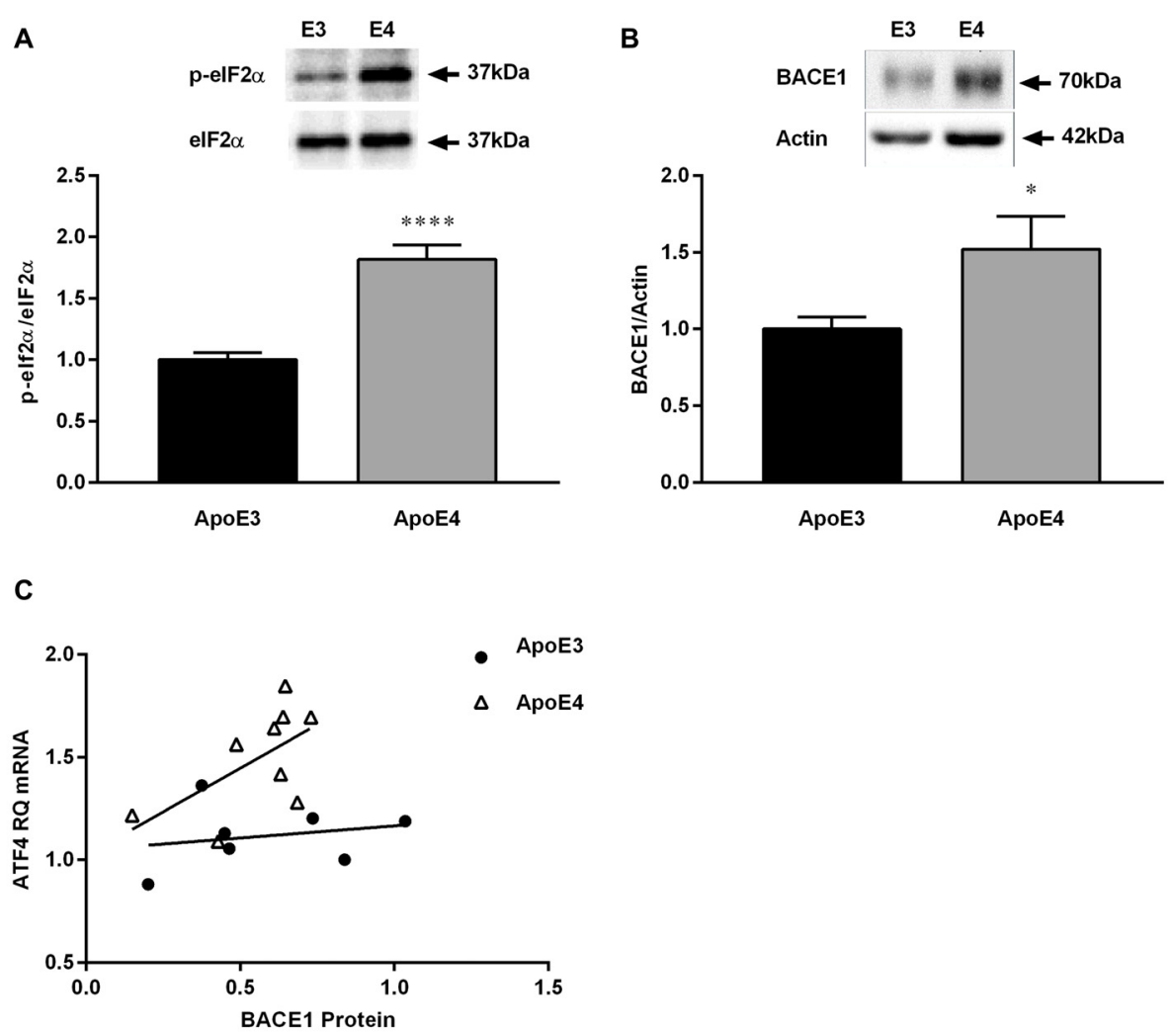

FIGURE 4 | HF diet promotes changes in $\beta$-site amyloid precursor protein-cleaving enzyme 1 (BACE1) protein expression in the hippocampus between ApoE3 and ApoE4 mice. Whole hippocampal tissue was punched from ApoE4 and ApoE3 mice after consuming a HF diet for 23 weeks, and analyzed for protein expression. (A) HF-ApoE4 retained basal-high levels of eukaryotic initiation factor 2 (elF2 $\alpha$ )-phosphorylation in the hippocampus (1.818 \pm 0.1171 , $n=14)$, compared to HF-ApoE3 mice (1.000 $\left.\pm 0.05911, n=15, t_{(27)}=6.367,{ }^{* * * *} p<0.0001\right)$. (B) HF diet induced increased protein levels of BACE1 in the hippocampus of HF-ApoE4 mice $(1.521 \pm 0.2154, n=14)$, compared with HF-ApoE3 mice $\left(1.000 \pm 0.07804, n=13, t_{(25)}=2.204,{ }^{*} p=0.0369\right)$. (C) HF-ApoE4 mice show a stronger tendency to correlation of hippocampal BACE1 protein with amygdala ATF4 mRNA levels $(p=0.0920)$, compared with HF-ApoE3 mice $(p=0.6335)$, using the Pearson $r$ test. Furthermore, differences between elevations are highly significant $\left(F_{(1,13)}=13.4541, p=0.002838\right)$.

increased body weight. It should be noted that due to the fact that in the current study normal-diet fed mice were not evaluated, we cannot exclude the possibility that changes seen in the different ApoE strains were the result of changes in the ApoE3 mice and not the ApoE4 mice. For this, more groups should be included. However, existing data from previous publications, as well as metabolic data obtained from Harlan Laboratories in C57BL/6 mice, suggest that the changes are indeed specific to the E4 allele.

Genetic association studies and meta-analysis suggest an association of BACE1 and ApoE4 to impose additional risk for sporadic AD (Clarimón et al., 2003; Gold et al., 2003; Kan et al., 2005; Jo et al., 2008). In addition, studies suggest a possible role of saturated fatty acids as well as DM in the increased expression of BACE1; one group showed that conditioned medium from PA-treated astrocytes, caused BACE1 upregulation in cortical neurons (Patil et al., 2006; Zhang et al., 2009). Another group suggested that type 2 diabetes exacerbates the generation of A-beta via activation of BACE1 (Zhang et al., 2009). Importantly, we found HF treated ApoE4 mice possess increased protein levels of BACE1, the rate limiting enzyme in APP cleavage to $A \beta$, in the hippocampus, compared with HF-ApoE3 mice. We have never been able before to detect changes in BACE1 levels in normal diet-fed ApoE3 and ApoE4 mice (data not shown). Therefore, although the question of whether BACE1 levels are decreased in ApoE3 mice or increased in ApoE4 mice should be further studied using additional normal-diet fed groups, our previous results and the literature regarding BACE1 and obesity suggest that the changes are indeed specific to the E4 allele. Kim et al. (2011) showed that BACE1 is not altered in an allele specific manner in ApoE haploinsufficient mice, E3/E3 vs. E4/E4. This suggests that environmental factors such as nutrition in $\epsilon 4$ carriers affects biochemical factors closely related to the pathology of $\mathrm{AD}$. In fact, in APP transgenic mice, BACE1 expression and activity were increased at sites of focal inflammation before $A \beta$ deposition, suggesting inflammatory processes may directly promote local production of $\mathrm{A} \beta$ (Heneka et al., 2005).

In order to explicitly determine whether the changes in the ApoE strains were a result of the HF diet induced, further analyzing using additional groups of mice fed with a normal diet 
should be considered. However, we believe that essential and novel data could be concluded from our work here, which may open new horizons for studying $\mathrm{AD}$ progression in $\varepsilon 4$ carriers.

In regard to the ApoE4 genotype, our results and the literature suggest that chronic metabolic burden such as inflammation may result in increased BACE1 levels, and as a result, in accumulation of $A \beta$ peptides with time. In fact, it has been shown that ApoE4 promotes aggregation and amyloid plaque formation by displaying a weaker affinity for $A \beta$ peptides than other ApoE variants (Liu et al., 2007). The exact mechanism by which a HF diet in combination with ApoE4 induces metabolic stress and increased BACE1 levels should be further studied. The above also strengthens the hypothesis that $\mathrm{AD}$-related abnormality starts long before pathological features are apparent, and that these may be, to some extent, balanced by a healthy nutritional diet.

In the present work we demonstrate how the complex integration of genetic, metabolic and environmental factors triggers a vicious cycle having a detrimental effect on mental stress and cognitive state. In fact, environmental influences on longevity are of greatest importance. In rats, mice and nonhuman primates, caloric restriction prolongs life and retards the appearance of several conditions associated with aging (Bunout and Cambiazo, 1999). It would be intriguing to evaluate our new hypothesis that a balanced, healthy nutrition diet which meets the individual's needs in regards to specific genetic and

\section{REFERENCES}

Arbones-Mainar, J. M., Johnson, L. A., Altenburg, M. K., and Maeda, N. (2008). Differential modulation of diet-induced obesity and adipocyte functionality by human apolipoprotein E3 and E4 in mice. Int. J. Obes. 32, 1595-1605. doi: 10. 1038/ijo.2008.143

Bale, T. L., and Vale, W. W. (2004). CRF and CRF receptors: role in stress responsivity and other behaviors. Annu. Rev. Pharmacol. Toxicol. 44, 525-557. doi: 10.1146/annurev.pharmtox.44.101802.121410

Biessels, G. J., Kappelle, L. J., and Utrecht Diabetic Encephalopathy Study Group. (2005). Increased risk of Alzheimer's disease in Type II diabetes: insulin resistance of the brain or insulin-induced amyloid pathology? Biochem. Soc. Trans. 33, 1041-1044. doi: 10.1042/bst03 31041

Blanchard, D. C., Blanchard, R. J., Tom, P., and Rodgers, R. J. (1990). Diazepam changes risk assessment in an anxiety/defense test battery. Psychopharmacology (Berl) 101, 511-518. doi: 10.1007/bf022 44230

Bunout, D., and Cambiazo, V. (1999). Nutrition and aging. Rev. Med. Chil. 127, 82-88.

Caselli, R. J., Dueck, A. C., Osborne, D., Sabbagh, M. N., Connor, D. J., Ahern, G. L., et al. (2009). Longitudinal modeling of age-related memory decline and the APOE $\varepsilon 4$ effect. $N$ Engl. J. Med. 361, 255-263. doi: 10. 1056/NEJMoa0809437

Castellano, J. M., Kim, J., Stewart, F. R., Jiang, H., DeMattos, R. B., Patterson, B. W., et al. (2011). Human apoE isoforms differentially regulate brain amyloid$\beta$ peptide clearance. Sci. Transl. Med. 3:89ra57. doi: 10.1126/scitranslmed. 3002156

Chang, R. C., Wong, A. K., Ng, H. K., and Hugon, J. (2002). Phosphorylation of eukaryotic initiation factor- $2 \alpha(\mathrm{eIF} 2 \alpha)$ is associated with neuronal degeneration in Alzheimer's disease. Neuroreport 13, 2429-2432. doi: 10.1097/00001756200212200-00011

Chen, H. K., Liu, Z., Meyer-Franke, A., Brodbeck, J., Miranda, R. D., McGuire, J. G., et al. (2012). Small molecule structure correctors abolish detrimental metabolic profile, may delay some of the early symptoms of $\mathrm{AD}$.

\section{AUTHOR CONTRIBUTIONS}

YS designed the study, performed the experiments, analyzed the data, and wrote the article. AL performed the experiments and analyzed the data. MM performed the experiments and analyzed the data. KR designed the study and wrote the manuscript.

\section{ACKNOWLEDGMENTS}

This work was supported by the Israeli Ministry of Science, Technology, and Space (MOST 3-12080) and Wolfson foundation grants to KR. We thank laboratory members of KR, specifically Dr. Shunit Gal Ben-Ari for critical reading of the manuscript and Ms. Tali Rosenberg for the statistical analysis. We thank Dr. Derek LeRoith, Director of "Diabetes and Metabolism Clinical Research Center of Excellence" and Ms. Zila Shen-Orr from the Clinical Research Institute at Rambam Health Care Campus for their help with insulin and glucose analysis. We thank Prof. Alon Chen and Mr. Assaf Ramot from the Neurobiology of Stress Laboratory, Faculty of Biology, Department of Neurobiology, Weizmann Institute of Science, for their help in CRF-system mRNA analysis.

effects of apolipoprotein E4 in cultured neurons. J. Biol. Chem. 287, 5253-5266. doi: 10.1074/jbc.m111.276162

Clarimón, J., Bertranpetit, J., Calafell, F., Boada, M., Tàrraga, L., and Comas, D. (2003). Association study between Alzheimer's disease and genes involved in $\mathrm{A} \beta$ biosynthesis, aggregation and degradation: suggestive results with BACE1. J. Neurol. 250, 956-961. doi: 10.1007/s00415-0031127-8

Costa-Mattioli, M., Gobert, D., Stern, E., Gamache, K., Colina, R., Cuello, C., et al. (2007). eIF2 $\alpha$ phosphorylation bidirectionally regulates the switch from shortto long-term synaptic plasticity and memory. Cell 129, 195-206. doi: 10.1016/j. cell.2007.01.050

Dacks, P. A., Andrieu, S., Blacker, D., Carman, A. J., Green, A. M., Grodstein, F. et al. (2014). Dementia prevention: optimizing the use of observational data for personal, clinical and public health decision-making. J. Prev. Alzheimers Dis. 1, $117-123$.

Dafnis, I., Tzinia, A. K., Tsilibary, E. C., Zannis, V. I., and Chroni, A. (2012). An apolipoprotein E4 fragment affects matrix metalloproteinase 9, tissue inhibitor of metalloproteinase 1 and cytokine levels in brain cell lines. Neuroscience 210, 21-32. doi: 10.1016/j.neuroscience.2012. 03.013

De Jager, P. L., Shulman, J. M., Chibnik, L. B., Keenan, B. T., Raj, T., Wilson, R. S., et al. (2012). A genome-wide scan for common variants affecting the rate of age-related cognitive decline. Neurobiol. Aging 33, 1017.e1-1017.e15. doi: 10. 1016/j.neurobiolaging.2011.09.033

de la Monte, S. M., and Wands, J. R. (2008). Alzheimer's disease is type 3 diabetes-evidence reviewed. J. Diabetes Sci. Technol. 2, 1101-1113. doi: 10. $1177 / 193229680800200619$

Devi, L., Alldred, M. J., Ginsberg, S. D., and Ohno, M. (2012). Mechanisms underlying insulin deficiency-induced acceleration of $\beta$-amyloidosis in a mouse model of Alzheimer's disease. PLoS One 7:e32792. doi: 10.1371/journal.pone. 0032792

Ferretti, L., McCurry, S. M., Logsdon, R., Gibbons, L., and Teri, L. (2001). Anxiety and Alzheimer's disease. J. Geriatr. Psychiatry Neurol. 14, 52-58. doi: 10 $1177 / 089198870101400111$ 
Fukumoto, H., Cheung, B. S., Hyman, B. T., and Irizarry, M. C. (2002). $\beta$-secretase protein and activity are increased in the neocortex in Alzheimer disease. Arch. Neurol. 59, 1381-1389. doi: 10.1001/archneur.59.9.1381

Gold, G., Blouin, J. L., Herrmann, F. R., Michon, A., Mulligan, R., Duriaux Sail, G., et al. (2003). Specific BACE1 genotypes provide additional risk for lateonset Alzheimer disease in APOE epsilon 4 carriers. Am. J. Med. Genet. B Neuropsychiatr. Genet. 119B, 44-47. doi: 10.1002/ajmg.b.10010

Griebel, G., Blanchard, D. C., Jung, A., and Blanchard, R. J. (1995). A model of 'antipredator' defense in Swiss-Webster mice: effects of benzodiazepine receptor ligands with different intrinsic activities. Behav. Pharmacol. 6, 732-745. doi: 10.1097/00008877-199511000-00009

Griebel, G., Rodgers, R. J., Perrault, G., and Sanger, D. J. (1997). Risk assessment behaviour: evaluation of utility in the study of 5-HT-related drugs in the rat elevated plus-maze test. Pharmacol. Biochem. Behav. 57, 817-827. doi: 10. 1016/s0091-3057(96)00402-9

Hartman, R. E., Wozniak, D. F., Nardi, A., Olney, J. W., Sartorius, L., and Holtzman, D. M. (2001). Behavioral phenotyping of GFAP-apoE3 and -apoE4 transgenic mice: apoE4 mice show profound working memory impairments in the absence of Alzheimer's-like neuropathology. Exp. Neurol. 170, 326-344. doi: 10.1006/exnr.2001.7715

Hauger, R. L., Risbrough, V., Brauns, O., and Dautzenberg, F. M. (2006). Corticotropin releasing factor (CRF) receptor signaling in the central nervous system: new molecular targets. CNS Neurol. Disord. Drug Targets 5, 453-479. doi: $10.2174 / 187152706777950684$

He, X., Cooley, K., Chung, C. H., Dashti, N., and Tang, J. (2007). Apolipoprotein receptor 2 and X11 $\alpha / \beta$ mediate apolipoprotein E-induced endocytosis of amyloid- $\beta$ precursor protein and $\beta$-secretase, leading to amyloid- $\beta$ production. J. Neurosci. 27, 4052-4060. doi: 10.1523/JNEUROSCI.3993-06.2007

Heneka, M. T., Sastre, M., Dumitrescu-Ozimek, L., Dewachter, I., Walter, J., Klockgether, T., et al. (2005). Focal glial activation coincides with increased BACE1 activation and precedes amyloid plaque deposition in APP [V717I] transgenic mice. J. Neuroinflammation 2:22. doi: 10.1186/1742-2094-2-22

Ho, L., Qin, W., Pompl, P. N., Xiang, Z., Wang, J., Zhao, Z., et al. (2004). Diet-induced insulin resistance promotes amyloidosis in a transgenic mouse model of Alzheimer's disease. FASEB J. 18, 902-904. doi: 10.1096/fj.030978fje

Hogg, S. (1996). A review of the validity and variability of the elevated plus-maze as an animal model of anxiety. Pharmacol. Biochem. Behav. 54, 21-30. doi: 10. 1016/0091-3057(95)02126-4

Huebbe, P., Dose, J., Schloesser, A., Campbell, G., Gluer, C. C., Gupta, Y., et al. (2015). Apolipoprotein E(APOE) genotype regulates body weight and fatty acid utilization-Studies in gene-targeted replacement mice. Mol. Nutr. Food Res. 59, 334-343. doi: 10.1002/mnfr.201400636

Jo, S. A., Ahn, K., Kim, E., Kim, H. S., Jo, I., Kim, D. K., et al. (2008). Association of BACE1 gene polymorphism with Alzheimer's disease in Asian populations: meta-analysis including Korean samples. Dement. Geriatr. Cogn. Disord. 25, 165-169. doi: 10.1159/000112918

Johnson, L. A., Olsen, R. H., Merkens, L. S., DeBarber, A., Steiner, R. D., Sullivan, P. M., et al. (2014). Apolipoprotein E-low density lipoprotein receptor interaction affects spatial memory retention and brain ApoE levels in an isoform-dependent manner. Neurobiol. Dis. 64, 150-162. doi: 10.1016/j.nbd. 2013.12.016

Kan, R., Wang, B., Zhang, C., Jin, F., Yang, Z., Ji, S., et al. (2005). Genetic association of BACE1 gene polymorphism C786G with late-onset Alzheimer's disease in chinese. J. Mol. Neurosci. 25, 127-131. doi: 10.1385/jmn:25:2:127

Kim, J., Jiang, H., Park, S., Eltorai, A. E., Stewart, F. R., Yoon, H., et al. (2011). Haploinsufficiency of human APOE reduces amyloid deposition in a mouse model of amyloid- $\beta$ amyloidosis. J. Neurosci. 31, 18007-18012. doi: 10. 1523/JNEUROSCI.3773-11.2011

Kornecook, T. J., McKinney, A. P., Ferguson, M. T., and Dodart, J. C. (2010). Isoform-specific effects of apolipoprotein $\mathrm{E}$ on cognitive performance in targeted-replacement mice overexpressing human APP. Genes Brain Behav. 9, 182-192. doi: 10.1111/j.1601-183X.2009.00545.x

Li, W. H., Tanimura, M., Luo, C. C., Datta, S., and Chan, L. (1988). The apolipoprotein multigene family: biosynthesis, structure, structure-function relationships and evolution. J. Lipid Res. 29, 245-271.

Liu, Q., Zerbinatti, C. V., Zhang, J., Hoe, H. S., Wang, B., Cole, S. L., et al. (2007). Amyloid precursor protein regulates brain apolipoprotein $\mathrm{E}$ and cholesterol metabolism through lipoprotein receptor LRP1. Neuron 56, 66-78. doi: 10. 1016/j.neuron.2007.08.008

Maesako, M., Uemura, M., Tashiro, Y., Sasaki, K., Watanabe, K., Noda, Y., et al. (2015). High fat diet enhances $\beta$-site cleavage of Amyloid Precursor Protein (APP) via Promoting $\beta$-Site APP cleaving enzyme 1/Adaptor protein 2/Clathrin complex formation. PloS One 10:e0131199. doi: 10.1371/journal.pone.0131199

Mahley, R. W., Rall, S. C. Jr. (2000). Apolipoprotein E: far more than a lipid transport protein. Annu. Rev. Genomics Hum. Genet. 1, 507-537. doi: 10. 1146/annurev.genom.1.1.507

Meakin, P. J., Harper, A. J., Hamilton, D. L., Gallagher, J., McNeilly, A. D., Burgess, L. A., et al. (2012). Reduction in BACE1 decreases body weight, protects against diet-induced obesity and enhances insulin sensitivity in mice. Biochem. J. 441, 285-296. doi: 10.1042/BJ20110512

O'Connor, T., Sadleir, K. R., Maus, E., Velliquette, R. A., Zhao, J., Cole, S. L., et al. (2008). Phosphorylation of the translation initiation factor eIF2 $\alpha$ increases BACE1 levels and promotes amyloidogenesis. Neuron 60, 988-1009. doi: 10. 1016/j.neuron.2008.10.047

Olsen, R. H., Agam, M., Davis, M. J., and Raber, J. (2012). ApoE isoformdependent deficits in extinction of contextual fear conditioning. Genes Brain Behav. 11, 806-812. doi: 10.1111/j.1601-183X.2012.00833.x

Page, G., Rioux Bilan, A., Ingrand, S., Lafay-Chebassier, C., Pain, S., Perault Pochat, M. C., et al. (2006). Activated double-stranded RNA-dependent protein kinase and neuronal death in models of Alzheimer's disease. Neuroscience 139, 1343-1354. doi: 10.1016/j.neuroscience.2006.01.047

Patil, S., Sheng, L., Masserang, A., and Chan, C. (2006). Palmitic acid-treated astrocytes induce BACE1 upregulation and accumulation of C-terminal fragment of APP in primary cortical neurons. Neurosci. Lett. 406, 55-59. doi: 10.1016/j.neulet.2006.07.015

Peila, R., Rodriguez, B. L., Launer, L. J., and Honolulu-Asia Aging Study. (2002). Type 2 diabetes, APOE gene and the risk for dementia and related pathologies: the honolulu-asia aging study. Diabetes 51, 1256-1262. doi: 10.2337/diabetes. 51.4.1256

Pendse, A. A., Arbones-Mainar, J. M., Johnson, L. A., Altenburg, M. K., and Maeda, N. (2009). Apolipoprotein E knock-out and knock-in mice: atherosclerosis, metabolic syndrome and beyond. J. Lipid Res. 50, S178-S182. doi: 10.1194/jlr.R800070-JLR200

Raber, J., Wong, D., Yu, G. Q., Buttini, M., Mahley, R. W., Pitas, R. E., et al. (2000). Apolipoprotein E and cognitive performance. Nature 404, 352-354. doi: 10. $1038 / 35006165$

Rall, S. C. Jr., Weisgraber, K. H., and Mahley, R. W. (1982). Human apolipoprotein E. The complete amino acid sequence. J. Biol. Chem. 257, 4171-4178.

Robertson, J., Curley, J., Kaye, J., Quinn, J., Pfankuch, T., and Raber, J. (2005). apoE isoforms and measures of anxiety in probable $\mathrm{AD}$ patients and Apoe/- mice. Neurobiol. Aging 26, 637-643. doi: 10.1016/j.neurobiolaging.2004. 06.003

Salomon-Zimri, S., Boehm-Cagan, A., Liraz, O., and Michaelson, D. M. (2014). Hippocampus-related cognitive impairments in young apoE4 targeted replacement mice. Neurodegener. Dis. 13, 86-92. doi: 10.1159/000354777

Schipper, H. M. (2011). Apolipoprotein E: implications for AD neurobiology, epidemiology and risk assessment. Neurobiol. Aging 32, 778-790. doi: 10.1016/j. neurobiolaging.2009.04.021

Segev, Y., Barrera, I., Ounallah-Saad, H., Wibrand, K., Sporild, I., Livne, A., et al. (2015). PKR inhibition rescues memory deficit and ATF4 overexpression in ApoE $\varepsilon 4$ human replacement mice. J. Neurosci. 35, 12986-12993. doi: 10. 1523/JNEUROSCI.5241-14.2015

Segev, Y., Michaelson, D. M., and Rosenblum, K. (2013). ApoE $\varepsilon 4$ is associated with eIF2 $\alpha$ phosphorylation and impaired learning in young mice. Neurobiol. Aging 34, 863-872. doi: 10.1016/j.neurobiolaging.2012. 06.020

Siegel, J. A., Haley, G. E., and Raber, J. (2012). Apolipoprotein E isoformdependent effects on anxiety and cognition in female TR mice. Neurobiol. Aging 33, 345-358. doi: 10.1016/j.neurobiolaging.2010.03.002

Son, S. M., Song, H., Byun, J., Park, K. S., Jang, H. C., Park, Y. J., et al. (2012). Accumulation of autophagosomes contributes to enhanced amyloidogenic APP processing under insulin-resistant conditions. Autophagy 8, 1842-1844. doi: 10.4161 /auto. 21861

Sonenberg, N., Hershey, J. W., and Mathews, M. (2000). Translational Control of Gene Expression. New York, NY: Cold Spring Harbor Laboratory Press. 
Strittmatter, W. J., and Roses, A. D. (1996). Apolipoprotein E and Alzheimer's disease. Ann. Rev. Neurosci. 19, 53-77. doi: 10.1007/978-3-642-80109-9_2

Ward, A., Crean, S., Mercaldi, C. J., Collins, J. M., Boyd, D., Cook, M. N., et al. (2012). Prevalence of apolipoprotein E4 genotype and homozygotes (APOE e4/4) among patients diagnosed with Alzheimer's disease: a systematic review and meta-analysis. Neuroepidemiology 38, 1-17. doi: 10.1159/000334607

Yang, Y., and Song, W. (2013). Molecular links between Alzheimer's disease and diabetes mellitus. Neuroscience 250, 140-150. doi: 10.1016/j.neuroscience.2013. 07.009

Zhang, T., Pan, B. S., Zhao, B., Zhang, L. M., Huang, Y. L., and Sun, F. Y. (2009). Exacerbation of poststroke dementia by type 2 diabetes is associated with synergistic increases of $\beta$-secretase activation and $\beta$-amyloid generation in rat brains. Neuroscience 161, 1045-1056. doi: 10.1016/j.neuroscience.2009. 04.032
Conflict of Interest Statement: The authors declare that the research was conducted in the absence of any commercial or financial relationships that could be construed as a potential conflict of interest.

The reviewer JVS-M and handling Editor declared their shared affiliation, and the handling Editor states that the process nevertheless met the standards of a fair and objective review.

Copyright (C) 2016 Segev, Livne, Mints and Rosenblum. This is an open-access article distributed under the terms of the Creative Commons Attribution License (CC BY). The use, distribution and reproduction in other forums is permitted, provided the original author(s) or licensor are credited and that the original publication in this journal is cited, in accordance with accepted academic practice. No use, distribution or reproduction is permitted which does not comply with these terms. 